\title{
10000 Balloon-assisted Catheterization Technical Technique under Proximal Flow Control with Balloon Guiding Catheter in Endovascular Treatment of Cerebral Aneurysm
}

Hiroaki Minami, ${ }^{1}$ Hiroaki Hanayama, ${ }^{1}$ Takashi Okada, ${ }^{1}$ Hiroaki Matsumoto, ${ }^{1}$ Yasuo Sakurai,,${ }^{1}$ Atsushi Masuda, ${ }^{1}$ Shogo Tominaga, ${ }^{1}$ Katsuya Miyaji, ${ }^{1}$ Ikuya Yamaura, ${ }^{1}$ Yutaka Hirata, ${ }^{2}$ and Yasuhisa Yoshida ${ }^{1}$

Objective: A compliant balloon microcatheter has been used conventionally to perform remodeling in the treatment of broad-neck intracranial aneurysms. We describe a balloon-assisted microcatheterization technique using a compliant balloon microcatheter under proximal flow control of a balloon guiding catheter for the purpose of microcatheter navigation into aneurysms with anatomical difficulty.

Case Presentations: We used this technique successfully in six patients who had non-ruptured paraclinoid aneurysms. A balloon guiding catheter is placed in the parent artery. A compliant balloon catheter is navigated to distal to the aneurysmal neck for occlusion of the distal route. After inflating the balloon of the guiding catheter, the compliant balloon is inflated. The distal balloon creates a barrier, and only the path to the aneurysm remains. Balloon inflation of the guiding catheter is helpful to stabilize a distal compliant balloon so that the balloon does not float. In addition, proximal flow control of the parent artery may prevent blood pressure from increasing inside the aneurysm and leading to aneurysm rupture when a compliant balloon is inflated distal to the aneurysmal neck.

Conclusion: If catheter detention to an aneurysm in coil embolization is anatomically hazardous, this method is easy, safe, and very useful, and an important option.

Keywords catheterization, compliant balloon, cerebral aneurysm, balloon guiding catheter, proximal flow control

\section{Introduction}

Navigation of the microcatheter is essential in endovascular procedures for the treatment of intracranial aneurysm. However, we sometimes encounter procedural difficulties with catheterization into the aneurysmal sac. An acute angle between the target and parent arteries, the projection

${ }^{1}$ Department of Neurosurgery, Eishokai Yoshida Hospital, Cerebrovascular Institute, Kobe, Hyogo, Japan

${ }^{2}$ Department of Neurology, Eishokai Yoshida Hospital, Cerebrovascular Institute, Kobe, Hyogo, Japan

Received: May 7, 2016; Accepted: October 15, 2016 Corresponding author: Hiroaki Minami. Department of Neurosurgery, Eishokai Yoshida Hospital, Cerebrovascular Institute, 9-2-6 Daikai-dori, Hyogo-ku, Kobe, Hyogo 652-0803, Japan

Email: h-minami@yoshida-hp.or.jp

(C)2017 The Editorial Committee of Journal of Neuroendovascular Therapy. All rights reserved. of aneurysms with unfavorable angle to parent vessels, and acute curves of parent arteries represent hazards to catheter navigation. Shaping of the microguidewire or steam shaping of a microcatheter is now common technique for interventional procedures ${ }^{1-3)}$ and often proved effective in selective catheterization of arteries originating with an acute angle. However, if such methods are not successful, operating time is greatly extended and the burden on operators is markedly increased. We have therefore performed balloon-assisted catheterization using a compliant balloon microcatheter as a technical option. A compliant balloon microcatheter has been utilized for endovascular coiling of wide-neck aneurysms as a remodeling technique. $\left.{ }^{4}\right)$ When our technique is attempted for endovascular coiling of aneurysms, proximal flow control by a balloon guiding catheter is used simultaneously. This is because balloon inflation on the guiding catheter is useful to stabilize a distal compliant balloon so that the balloon does not float and 
also prevent increases in blood pressure inside the aneurysm that might lead to aneurysm rupture when a compliant balloon is inflated distal to the aneurysmal neck. We have successfully applied this technique to treat paraclinoid carotid aneurysms with coil embolization. We propose that this technique can be performed safely and effectively.

\section{Methods}

From May 2012 to April 2016, a total of 116 patients with 118 intracranial aneurysms were treated endovascularly at our institute. Among these, 27 patients showed single paraclinoid aneurysms. Six aneurysms of these 27 patients were treated with coil embolization using a balloonassisted microcatheterization technique with a compliant balloon microcatheter under proximal flow control with a balloon guiding catheter.

\section{Technical description}

All patients were given $75 \mathrm{mg}$ of clopidogrel and $100 \mathrm{mg}$ of aspirin once a day for at least 7 days prior to treatment. VerifyNow (Accumetrics, San Diego, CA, USA) antiplatelet assays were performed before treatment to confirm the efficacy of these antiplatelet agents. Endovascular treatment was performed under general anesthesia and systemic heparinization. An $8-\mathrm{Fr} / 25-\mathrm{cm}$ sheath or $9-\mathrm{Fr} / 25-\mathrm{cm}$ sheath was inserted into the femoral artery. The tip of an 8-Fr or 9-Fr balloon guiding catheter (Optimo; Tokai Medical Products, Kasugai, Japan) was advanced coaxially and placed at the distal portion of the cervical carotid artery from the beginning. In the two most recent cases, a triple coaxial method ${ }^{5}$ was simultaneously adopted with insertion of a 6-Fr inner catheter (Cerulean; Medikit, Tokyo, Japan) through the balloon guiding catheter. The inner catheter was also advanced coaxially and the tip deployed to the intracranial portion of the carotid artery for much better stabilization of the microcatheter. Our maneuver can usually be accomplished using an 8-Fr Optimo, but a 9-Fr Optimo is necessary for the triple coaxial method. A compliant balloon microcatheter (Hyperglide/Hyperform; ev3/Covidian, Irvine, CA, USA or Scepter; Microvention/Terumo, Tustin, CA, USA) was navigated to distal to the aneurysm neck. After inflating the Optimo balloon to reduce blood flow, the balloon of the compliant balloon microcatheter was inflated. The compliant balloon should be expanded so that the end of the balloon is located slightly proximal to the distal end of the aneurysm neck to facilitate navigation of the microcatheter for coiling (Fig. 1). An artificial block by the inflated balloon is made

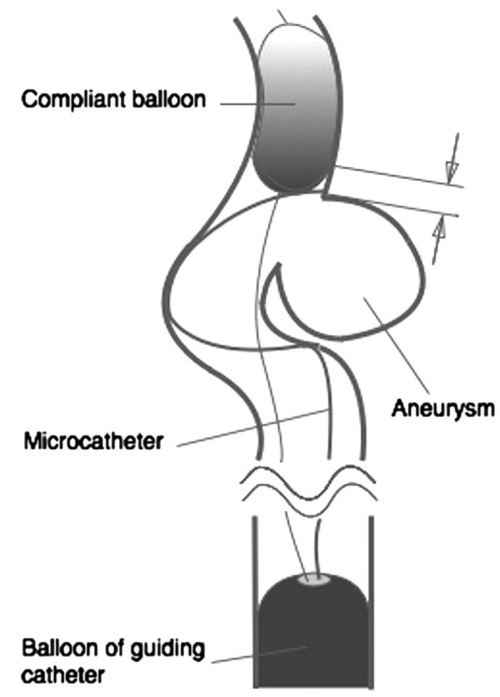

A

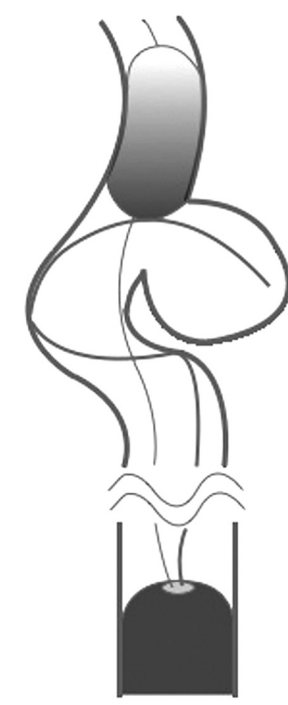

B
Fig. 1 (A) When the end of the balloon is located just distal to the aneurysmal neck, a microcatheter may not advance to the aneurysmal sac smoothly because of a gap between a compliant balloon and a parent artery (arrow). (B) The end of the balloon located slightly proximal to the distal end of the aneurysm neck can facilitate navigation of the microcatheter into the aneurysmal sac.

at the distal side of the parent artery of the aneurysm. As a result, only the path to the aneurysm remains open in this procedure. The microcatheter changed its orbit by the obstacle can then be easily guided into the aneurysm over the sufficiently curved microguidewire. Expansion of the balloon on the guiding catheter not only prevents the movement of the compliant balloon by arterial flow, but also may contribute to prevent increase in blood pressure in the aneurysm. Following microcatheter navigation to the aneurysm, the compliant balloon is deflated first, followed by the balloon of the guiding catheter. The compliant balloon microcatheter is retained and used for remodeling as needed.

\section{Results}

Demographic and clinical characteristics of the six patients are presented in Table 1.

Patients comprised five women $(83.3 \%)$ and one man (16.7\%). The mean age of patients was 62.1 years (range: 53-68 years). All six patients had unruptured aneurysms. With regard to aneurysmal location, four aneurysms were located in the carotid cave, one at the origin of the ophthalmic artery, and one at the origin of the superior hypophyseal artery. Regarding the angle between the aneurysmal dome projection and the parent artery on three-dimensional images obtained from angiography, an acute angle $\left(<90^{\circ}\right)$ was 


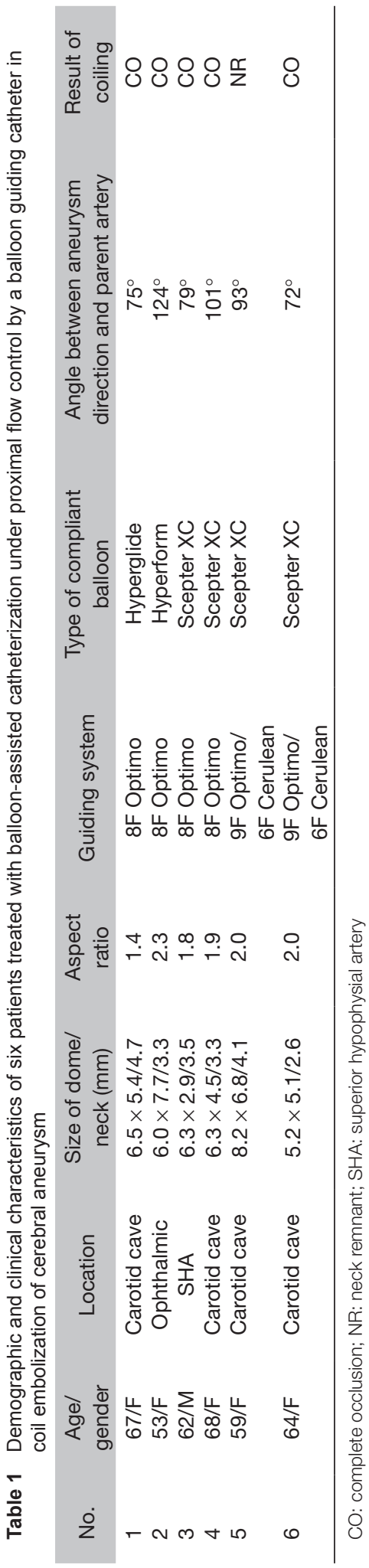

recognized in three cases. Maximum angle was $124^{\circ}$ in the patient with an ophthalmic artery aneurysm projecting superomedially. All aneurysms were larger than $5 \mathrm{~mm}$ in diameter. Four aneurysms had a narrow neck less than $4 \mathrm{~mm}$. The aspect ratio (depth/neck width) was larger than 1.8 in five cases. Successful catheterization to the aneurysm and coil embolization was achieved in all procedures, with complete aneurysm occlusion in $83.3 \%$, and neck remnants in $16.7 \%$. This maneuver was performed from the beginning in four cases, and in two cases started at the time when usual navigation of the microcatheter was unsuccessful. The exchange of a guiding catheter system was not necessary in all cases because we usually utilize a balloon guiding catheter in coil embolization of anterior circulation aneurysms for the purpose of temporary hemostasis for aneurysm rupture and stabilization of a compliant balloon catheter. The remodeling technique with the detained compliant balloon was utilized in all cases. No perioperative or postoperative complications were encountered. Diffusion-weighted imaging after coiling revealed a small ischemic lesion in one patient, but that patient presented with no neurological deficits.

\section{Case Presentations}

\section{Case 1}

A 53-year-old woman presenting with numbness in the left upper extremity was treated with endovascular coiling of a $6.0 \times 7.7-\mathrm{mm}$ carotid-ophthalmic artery aneurysm projecting superomedially (Figs. 2A-2C). Under general anesthesia, an 8-Fr sheath was placed in the right common femoral artery. Heparin was administered as a bolus of $5000 \mathrm{U}$, with additional boluses given as needed to maintain an activated clotting time $>250$ s. An 8-Fr Optimo balloon guiding catheter was inserted into the right cervical internal carotid artery. Attempts to navigate the microcatheter into the aneurysmal sac failed, so balloon-assisted microcatheterization under proximal flow control was performed. A HyperForm compliant balloon catheter was navigated to slightly proximal to the distal end of the aneurysm neck over a microguidewire (X-pedion; Micro Therapeutics, Irvine, CA, USA). The balloon of the guiding catheter and the compliant balloon were inflated, then a steam-shaped microcatheter (Excelsior SL-10; Stryker Neurovascular, Fremont, CA) was turned over a microguidewire (Tenrou; Japan Lifeline Corporation, Tokyo, Japan) and introduced into the aneurysmal sac smoothly within a short time (Figs. 2D and 2E). Each balloon was deflated. Coiling of the aneurysm was successfully accomplished using the remodeling technique and complete 


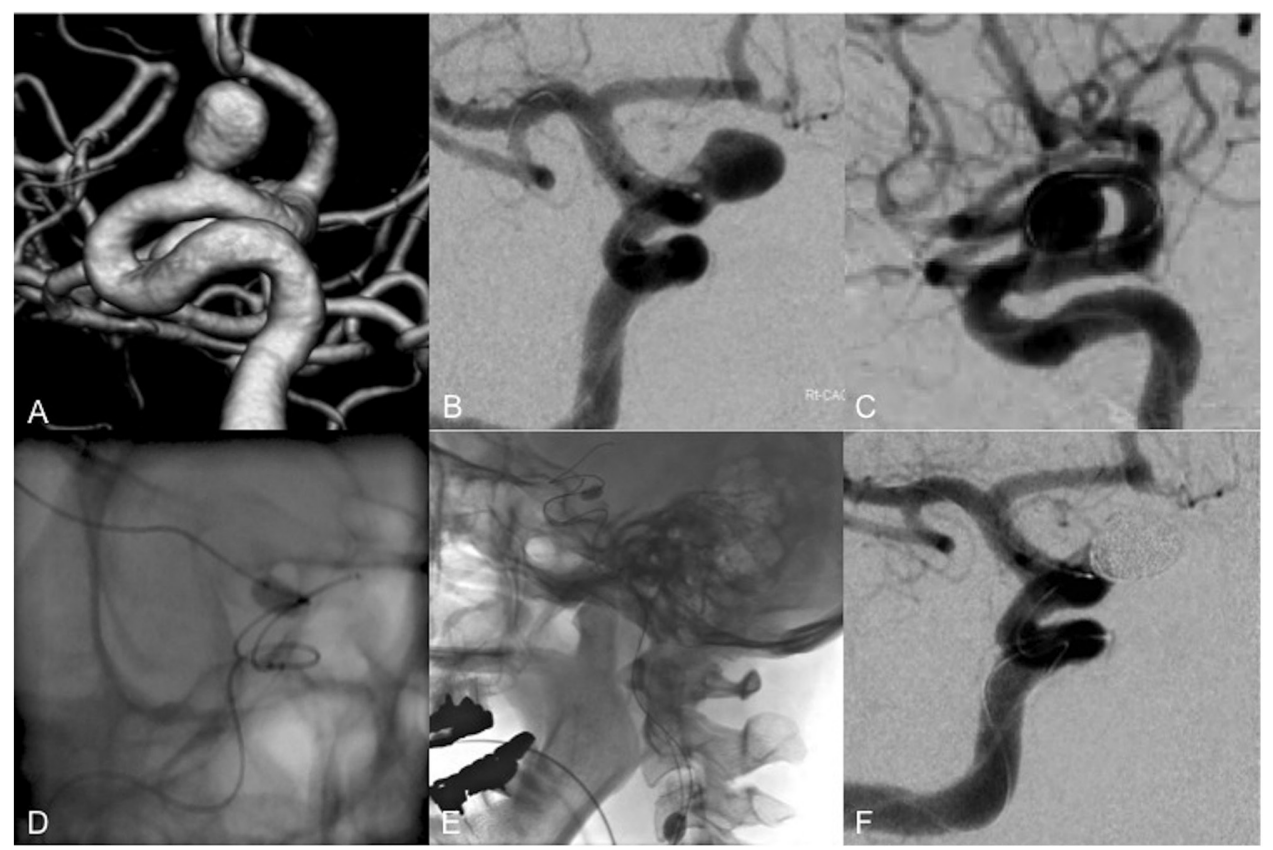

Fig. 2 Three-dimensional image (A), anteroposterior (B), and lateral view (C) of right carotid angiography reveal a carotid-ophthalmic artery aneurysm projecting superomedially. A microcatheter was introduced into the aneurysmal sac over a microguidewire, assisted by an inflated compliant balloon (D) under proximal flow control with the balloon of the guiding catheter (E). Complete occlusion of the aneurysm was obtained by coil embolization (F).

occlusion was obtained (Fig. 2F). The sheath was removed $4 \mathrm{~h}$ after the last dose of heparin was given. Femoral hemostasis was accomplished with manual compression. No procedural complications were identified.

\section{Case 2}

A 64-year-old woman with a history of chronic obstructive pulmonary disease presented with dizziness and was incidentally discovered to have an unruptured right-side carotid cave aneurysm (Fig. 3A). The patient was treated with endovascular coiling. Under general anesthesia, a 9-Fr sheath was placed in the right common femoral artery. Heparinization was initiated routinely. A 9-Fr Optimo balloon guiding catheter was placed at the right cervical internal carotid artery. A 6-Fr/ $115-\mathrm{cm}$ Cerulean with a 4-Fr/ $135-\mathrm{cm}$ Cerulean inner catheter was advanced over a guidewire through the Optimo to the $\mathrm{C} 4$ segment of the right carotid artery. The 4-Fr Cerulean was then removed. A triple coaxial system was thus established. Aneurysm size was $5.2 \times 5.1 \mathrm{~mm}$, and neck size was $2.6 \mathrm{~mm}$. The angle between the aneurysmal dome projection and the parent artery was $80^{\circ}$. We decided to perform balloon-assisted catheterization because difficulty with catheterization was anticipated on the basis of the aneurysm location and sharp angle. A compliant balloon catheter (Scepter $\mathrm{XC}$; Microvention/Terumo) was delivered through the 6-Fr
Cerulean over a microguidewire (Transend EX; Stryker Neurovascular, Fremont, CA) to slightly proximal to the distal end of the aneurysm neck. A microcatheter (Excelsior SL-10, preshaped J; Stryker Neurovascular) over a microguidewire (Asahi Chikai 10; Asahi Intecc, Nagoya, Japan) shaped in a gentle curve of $180^{\circ}$ through the 6-Fr Cerulean was advanced to just proximal to the aneurysmal neck. Each balloon of the Optimo and Scepter XC was inflated. The part of the loop of the microguidewire was affected by the inflated compliant balloon, the direction of the tip of the microguidewire was changed, and the microguidewire was carefully positioned within the aneurysmal sac. The microcatheter was then gently but easily navigated into the aneurysm over the microguidewire (Figs. 3B-3E). After each balloon was deflated, the Scepter XC was pulled back to a position covering the aneurysmal neck. Coil embolization was successfully performed using the remodeling technique (Fig. 3F). Manual compression for hemostasis was performed subsequent to removal of the femoral sheath. After treatment, the patient awoke neurologically intact and was discharged the following day.

\section{Discussion}

Navigation of the microcatheter is essential in endovascular procedures. In coil embolization of intracranial aneurysms, 


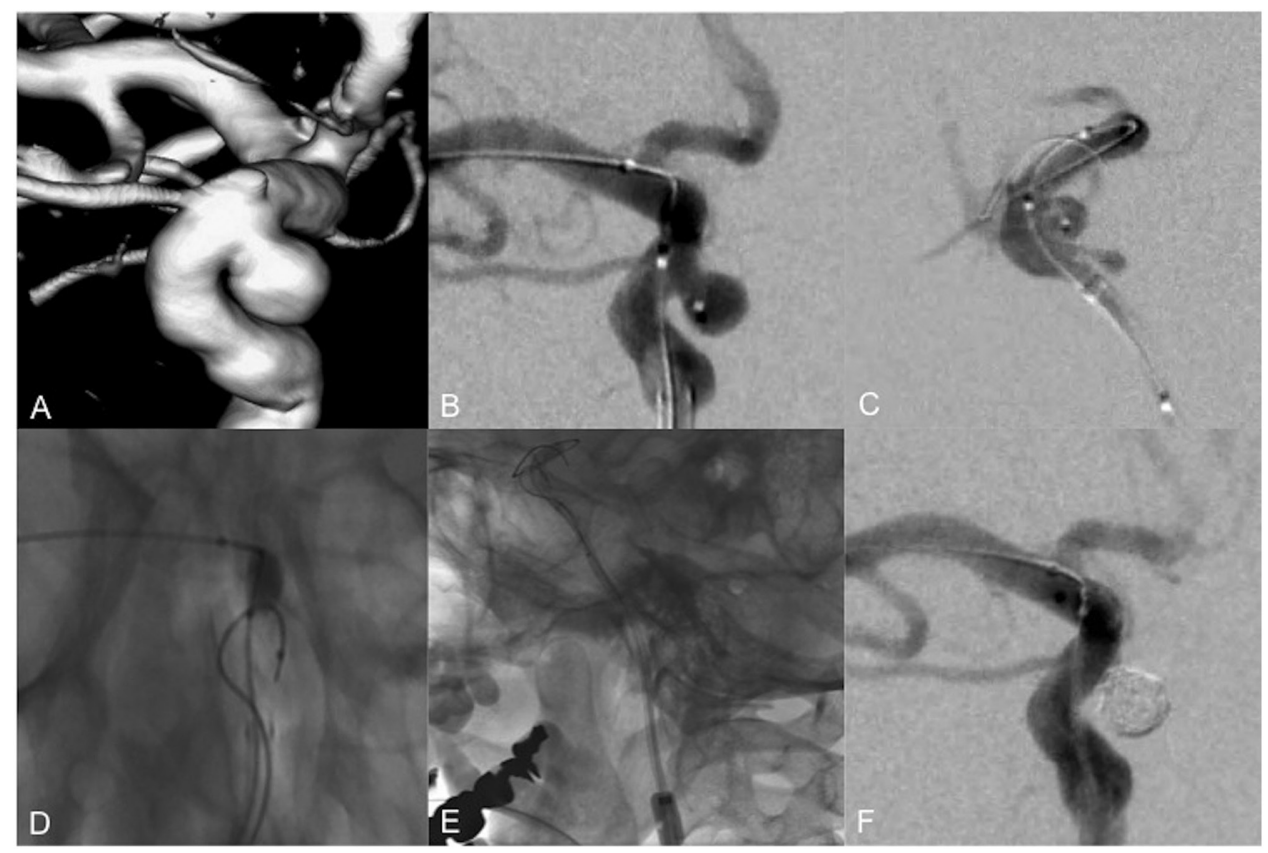

Fig. 3 (A) Three-dimensional image from right carotid angiography reveals a carotid-cave aneurysm projecting inferomedially with an acute angle between the axis of the aneurysm and the parent artery. A microcatheter was introduced into the aneurysmal sac over a microguidewire, assisted by the inflated compliant balloon (B-D) under proximal flow control with the balloon of the guiding catheter (E). Complete occlusion of the aneurysm was confirmed after the procedure $(\mathbf{F})$.

introduction of a microcatheter into the aneurysmal sac is definitely necessary. However, this sometimes proves difficult or even impossible because of the location, the projection of the aneurysm at an acute angle from the parent vessel, or tortuosity of the parent artery. Steam shaping of the microcatheter tip is frequently used in such cases. ${ }^{1,3)}$ Steam shaping is not only important for catheter insertion into aneurysms, but it also allows stabilization of a catheter during coil filling after insertion. ${ }^{2)}$ We have often utilized steam shaping of microcatheters or a preshaped microcatheter in coil embolization of aneurysms. However, such attempts sometimes result in failure, and even those that are possible may require a long time. Therefore, in cases with difficult catheterization for endovascular coiling of aneurysms, we have been performing balloon-assisted catheterization using a compliant balloon microcatheter under proximal flow control by a balloon guiding catheter. This maneuver is very simple and allows easy changing of the catheter direction by blocking alternative routes using a compliant balloon. This maneuver may facilitate catheterization into an acute branch as well as access into aneurysms. When this maneuver is performed, proximal flow control is useful to stabilize the compliant balloon and prevents flotation. In addition, proximal flow control offers the additional advantage of temporary hemostasis if aneurysm rupture occurs. Moreover, simply inflating a compliant balloon at distal to the aneurysmal neck may cause higher hemodynamic force on the aneurysmal wall at least temporarily, elevate arterial blood pressure inside aneurysms, and increase the risk of aneurysmal rupture although blood can decompress out the ophthalmic artery, cavernous branches, and the external carotid artery in some degree in paraclinoid lesions. We therefore add proximal flow control with this maneuver. Some reports have described microcatheter navigation using a compliant balloon in the endovascular treatment of arteriovenous malformation ${ }^{6}$ or dural arteriovenous fistula. ${ }^{78}$ However, we could not find any reports of balloon-assisted catheterization of aneurysms with simultaneous use of proximal flow control. Therefore, this appears to be the first report of this method.

Some technical tips will facilitate this procedure. The first involves appropriate inflation of the compliant balloon. If insufficient expansion of the balloon is achieved, the microguidewire and microcatheter may enter the interstices between the balloon and parent artery. Conversely, overinflation may cause dissection or rupture of the parent artery. The second tip involves positioning of the compliant balloon. Slightly proximal to just distal to the aneurysmal neck represents the optimal position for maximum ease of catheterization. The third is to avoid hitting the compliant balloon 
with the tip of the microguidewire. If the tip of the microguidewire comes in contact with and catches on the balloon surface, sudden advancement or perforation of the balloon may result. Shaping a suitable curve to the microguidewire, preferably in a U-turn shape, may prevent such complications.

In our small series, all aneurysms to which this technique was applied were located in a paraclinoid portion of the carotid artery. Difficulty with insertion and stabilization of microcatheter tips in aneurysms has been reported for coil embolization of paraclinoid aneurysms. ${ }^{9)}$ The anatomical characteristic of paraclinoid aneurysms being located just distal to the carotid siphon may cause difficulties in catheter navigation. It goes without saying that the maneuver we describe can be utilized for aneurysms in other locations. However, proximal flow control in this procedure is not applicable to anterior communicating artery aneurysms with blood supply through the contralateral A1 or for aneurysms in the posterior circulation other than vertebral artery aneurysms. This is because a guiding balloon in these aneurysms would not interrupt blood supply from the contralateral artery. With regard to morphology, medium size and larger aspect ratio (i.e. narrow neck sizes for constant depth) in addition to an acute angle were characteristics of aneurysms in our series. It is thought that this does not mean our maneuver is not suitable for wideneck aneurysms, but it reflects that procedural difficulties with catheterization in wide-neck aneurysms are rare. And also this maneuver could be applied to even small aneurysms with smaller aspect ratio, but it is necessary to care for sudden advancement of a microcatheter.

Some disadvantages of this maneuver must be borne in mind. One is that an 8-Fr or larger profile for the balloon guiding catheter is required for insertion of two microcatheters. In addition, sufficient administration of antiplatelet agents is necessary to prevent thromboembolic events. If possible, platelet aggregation should be confirmed before treatment. Vessel rupture or arterial dissection on balloon inflation should also be prevented by performing the procedure with care. No complications were encountered in any of the six cases treated using this procedure. In limited cases, this maneuver is safe and useful.

\section{Conclusion}

This balloon-assisted microcatheterization technique under proximal flow control with a balloon guiding catheter was safe, feasible, and very useful for introducing a catheter into an aneurysmal sac with a difficult access route. This technique appears to have potential for use as an option in hazardous catheterizations for coil embolization of cerebral aneurysms.

\section{Disclosure Statement}

The authors report no conflict of interest concerning the materials or methods used in this study or the findings specified in this paper.

\section{References}

1) Abe $T$, Hirohata M, Tanaka N, et al: Distal-tip shapeconsistency testing of steam-shaped microcatheters suitable for cerebral aneurysm coil placement. AJNR Am J Neuroradiol 2004; 25: 1058-1061.

2) Abe T, Hirohata M, Tanaka N, et al: Stability of microcatheter for cerebral aneurysm embolization after steam shaping. Interv Neuroradiol 2004; 10 Suppl 1: 117-120.

3) Kiyosue H, Hori Y, Matsumoto S, et al: Shapability, memory, and luminal changes in microcatheters after steam shaping: a comparison of 11 different microcatheters. AJNR Am J Neuroradiol 2005; 26: 2610-2616.

4) Kulcsar Z, Yilmaz H, Bonvin C, et al: Multiple coaxial catheter system for reliable access in interventional stroke therapy. Cardiovasc Intervent Radiol 2010; 33: 1205-1209.

5) Mendes GA, Silveira EP, Saleme S, et al: Balloon-assisted microcatheter navigation for AVM embolization: technical note. J Neurosurg 2015; 123: 1120-1124.

6) Moret J, Cognard C, Weill A, et al: The "Remodelling Technique" in the treatment of wide neck intracranial aneurysms. angiographic results and clinical follow-up in 56 cases. Interv Neuroradiol 1997; 3: 21-35.

7) Toyota S, Fujimoto Y, Iwamoto F, et al: Technique for shaping microcatheter tips in coil embolization of paraclinoid aneurysms using full-scale volume rendering images of 3D rotational angiography. Minim Invasive Neurosurg 2009; 52: 201-203.

8) van Rooij WJ, Sluzewski M, Beute GN: Tentorial artery embolization in tentorial dural arteriovenous fistulas. Neuroradiology 2006; 48: 737-743.

9) Zhao WY, Krings T, Yang PF, et al: Balloon-assisted superselective microcatheterization for transarterial treatment of cranial dural arteriovenous fistulas: technique and results. Neurosurgery 2012; 71: 269-273. 